УДК 622.692.48

О ТЕСТИРОВАНИИ АЛГОРИТМОВ ОБНАРУЖЕНИЯ УТЕЧЕК НА ОСНОВЕ ФУНКЦИЙ ЧУВСТВИТЕЛЬНОСТИ

\title{
ABOUT THE TESTING OF THE LEAK DETECTING ALGORITHMS BASED ON THE SENSITIVITY FUNCTION
}

Бондарь Д.В., Жолобов В.В., Варыбок Д.И., Надежкин О.С.

Научно-исследовательский институт трубопроводного транспорта, г. Москва, Российская Федерация

\author{
D.V. Bondar, V.V. Zholobov, D.I. Varybok, O.S. Nadezhkin
}

Transneft Research Institute for Oil and Oil Products Transportation, Moscow, Russian Federation

e-mail: zholobovvv@niitnn.transneft.ru

Аннотация. Для оценки влияния различных факторов на идентификацию параметров утечки применено аналитическое решение задачи об изотермической утечке из участка остановленного трубопровода. Функция давления, полученная для утечки через отверстие с заданными параметрами (решение прямой задачи), принята в качестве кривой давления при идеальных экспериментальных измерениях.

Цель настоящей работы - исследовать процесс восстановления параметров утечки (обратная задача) c помощью метода функций чувствительности. Указанным методом основной вариант обратной задачи решен полностью аналитически, часть вариантов доведена до системы конечных нелинейных соотношений. Исследовано поведение метода и его реализующего алгоритма в случаях, когда измерения отличаются от идеальных. Для этого в исходное аналитическое решение внесены различного вида возмущения. Рассмотрены такие факторы, как величина 
площади отверстия (расход) утечки, высотная отметка сечения утечки, величина временного интервала, на котором осуществляется идентификация, системные ошибки и периодические возмущения замеров давления в пределах погрешности датчиков, a также алгоритмы предварительной обработки исходной информации. Получено аналитическое представление погрешности восстановления параметров утечки и рассмотрены особенности сходимости алгоритма. Установлено существование минимально необходимого временного интервала, начиная с которого идентификация по рассматриваемому методу становится возможной, а также существование пределов достижимой точности идентифицированных параметров. Приведено несколько тестов, позволяющих получать решение некоторых обратных задач в аналитической форме. Отличительные особенности предлагаемых тестов заключаются в возможности получения аналитических выражений погрешности одновременного восстановления нескольких (более двух) параметров утечки.

Abstract. For the assessment of the influence of different uncontrolled variable on the identification of runoff data analytical solution of isothermal runoff from the stopped pipeline section was used. The pressure function which had been got for the runoff through the hole with preset parameter (solution of the direct problem) is taken as a pressure curve under optimum experimental measurements.

The aim of this work is to investigate the process of the runoff parameter recovery (inverse problem) with the help of the sensitivity function method. With this method the main variant of the indirect is solved analytically, some other variants are led to the system of finishing nonlinear relationships. The behavior of the method and the algorithm of its usage in cases when the measurements differ from the optimum measurements were investigated. For this purpose, in the initial analytical solution several types of perturbing effects were included. Such factors as the measure of the runoff's hole, the leakage 
consumption, elevation point, system mistakes, periodic perturbing effects of pressure measurements within the measurements accuracy of sensors and algorithms of prior information handling.

The analytical definition of inaccuracy of the leakage parameter recovery was received and special aspects of algorithm convergence were analyzed. We determined the existence of the minimum time interval necessary for starting the identification based on this method and the existence of limits of anticipated accuracy of identified parameters. Some tests allowing getting the solution of some inverse problems in analytical form are given below. Distinctive features of the given tests involve the opportunity to receive analytic expressions of inaccuracy of simultaneous recovery of some (more than two) parameters of the runoff or leakage.

Ключевые слова: аналитическое решение, обратная задача, параметры утечки, целевая функция, метод наименьших квадратов, минимум функционала, метод функций чувствительности, идентификация, итерационный процесс, сходимость, тестирование.

Key words: analytical solution, an inverse problem, the runoff parameter, objective function, least-squares method, minimum functional, sensitivity function method, identification, iterative process, convergence, testing.

\section{Введение}

Необходимость решения задач, связанных с утечками и несанкционированными отборами, вынуждает не только применять известные методы, но и разрабатывать новые подходы или их элементы без строгого математического обоснования. К теоретическому исследованию процесса утечки возможны различные подходы, требующие большого объёма экспериментальной информации. При этом все данные (экспериментальные) должны быть получены из одного опыта во 
избежание расхождений, неизбежных при попытке согласования результатов, полученных из отдельных опытов. До тех пор, пока не проведено фундаментального изучения физических процессов, протекающих при утечке, ставить вопрос о полном моделировании всех стадий утечки и априорном прогнозировании ее параметров с высокой точностью преждевременно. Необходимость количественных расчетов ставит задачу рационального сочетания методов математического и физического моделирований и рассмотрения обратных задач. Правильность применения математической технологии организации вычислительного алгоритма (включая и решение обратных задач) должна быть проверена на количественных тестах [1]. К ним относится сравнение прямых численных данных по характеристикам процесса с аналитическими решениями и с измеренными (экспериментальными) характеристиками реальных процессов. Построение аналитических тестов является важной составной частью процесса выявления погрешности при решении численного аналога математической модели (решении прямой задачи). Тестирование численного метода решения, в свою очередь, является лишь необходимым элементом проверки степени соответствия принятой (разработанной) математической модели реальному процессу.

Цель данной работы - не вопросы адекватности математической модели и не точности решения ее численного аналога, a анализ погрешности одного из численных методов решения на eе основе обратных задач. Поэтому структура аналитического решения прямой задачи должна давать возможность проведения в аналитической форме вычислений (полностью или частично) в соответствии с анализируемым алгоритмом решения обратной задачи. Возможные упрощения детерминированных моделей, даже достаточно далекие от реальности, дают, тем не менее, хорошую возможность для тестирования алгоритмов идентификации на их аналитических решениях. Имея в виду, что результаты анализа эффективности алгоритмов параметрической 
идентификации, полученные для моделей упрощенных, будут справедливы и для более сложных случаев, рассмотрен простейший вариант восстановления характеристик изотермической утечки на остановленном участке трубопровода.

\section{Расчетные соотношения}

Предположим, что плотность жидкости $\rho$ и внутренняя площадь поперечного сечения упругого трубопровода $f$ связаны с давлением следующими соотношениями (закон Гука):

$$
\begin{gathered}
\rho=\rho_{0}\left(1+\frac{p-p^{o}}{\mathrm{~K}_{g}}\right) ; \\
f=f_{0}\left(1+\frac{d}{\delta} \cdot \frac{p-p^{o}}{\mathrm{E}}\right),
\end{gathered}
$$

где $\rho$ - плотность жидкости;

$p$ - давление;

$\mathrm{K}_{g}$ - модуль сжатия жидкости (для нефти $\mathrm{K}_{g}=1.3 \div 1.4 \cdot 10^{9} \mathrm{H} / \mathrm{m}^{2}$ );

$d$ - диаметр трубопровода;

$\delta$ - толщина стенки трубы;

E - модуль упругости материала стенок трубы, величина которого для стальных труб $\mathrm{E}=1.8 \div 2 \cdot 10^{11} \mathrm{H} / \mathrm{m}^{2}$;

нижний индекс 0 относится к соответствующим параметрам при давлении $p=p^{o}$.

Приведенные соотношения представляют собой первые два члена разложения экспоненты в ряд по степеням, поэтому наряду с (1), (2) целесообразно в дальнейшем их применение в форме [2]

$$
\rho=\rho_{0} \exp \left[\alpha\left(p-p^{o}\right)\right] ; \quad, f=f_{0} \exp \left[\beta\left(p-p^{o}\right)\right],
$$




$$
\alpha=\frac{1}{\mathrm{~K}_{g}} ; \quad \beta=\frac{d}{\delta \cdot E}
$$

Величины $\alpha, \beta$ связаны со скоростью распространения волн давления в упругом трубопроводе $c$ (выявленной Н.Е. Жуковским) следующим образом:

$$
\alpha+\beta=\frac{1}{\rho_{0} c^{2}}
$$

где $c$ - скорость звука (определяется упругими свойствами стенок трубопровода и жидкости).

Используя (3) легко производить аналитические преобразования, разлагая экспоненту в ряд и удерживая первые два слагаемых лишь на последнем этапе (только в итоговой зависимости).

Производительность утечки $q_{*}$ определяем следующим образом

$$
q_{*}=\mu s_{*} \sqrt{2 \rho\left(p_{z=z_{*}}-p_{a t m}\right)}
$$

где $\mu$ - коэффициент расхода $(\mu=0.62)$;

$s_{*}-$ площадь отверстия утечки;

$p_{a t m}-$ атмосферное давление;

$Z_{*}{ }^{-}$высотная отметка сечения утечки на остановленном участке трубопровода.

При проведении расчетов значения величин $s_{*}, Z_{*}$ являются параметрами утечки, подлежащих определению. Критерий точности их определения, как правило, задают по методу наименьших квадратов

$$
I\left(s_{*}, Z_{*}\right)=\sum_{i}\left(p_{i}^{z m}-p_{i}\right)^{2}
$$


где $p_{i}^{z m}$ - измеренное;

$p_{i}-$ расчетное значение давления в точках $t_{i}$.

Формула (5) должна быть приведена к высотной отметке $z_{d}$ сечения, в котором находится датчик $\mathrm{c}$ помощью известного соотношения гидростатики:

$$
z_{d}-z_{*}+\left(p_{d}-p_{z=z_{*}}\right) / \rho_{0} g ; \quad p_{d}=p^{z m},
$$

где индекс $d$ относится к параметрам в сечении расположения датчика.

Если измерение задано в виде непрерывной кривой давления, то при аналитических выкладках целесообразно использование критерия (6) в интегральной форме:

$$
I\left(s_{*}, Z_{*}\right)=\int_{0}^{t}\left(p^{z m}-p\right)^{2} d t
$$

Реже используют метод наименьших модулей, который позволяет эффективно обрабатывать данные с аномально большими ошибками. Особенностью этого способа является то, что если ошибки наблюдений распределены не по нормальному закону, то оценки метода наименьших модулей могут быть эффективнее оценок метода наименьших квадратов, и можно ожидать более высокую точность определяемых параметров [3]. Целевая функция метода наименьших модулей имеет вид:

$$
I\left(s_{*}, Z_{*}\right)=\sum_{i}\left|p_{i}^{Z m}-p_{i}\right|
$$

Рассмотрим процедуру определения параметров аварийной утечки $s_{*}, Z_{*}$ на остановленном трубопроводе по кривой падения давления, полученной в процессе распрессовки (предполагается, что во всех 
сечениях участка $\left.p(t)>p_{a t m}\right)$. Задача идентификации параметров утечки с использованием данных измерений относится к классу обратных [4].

\section{Прямая задача утечки}

Баланс массы жидкости на остановленном участке может быть записан в следующей форме:

$$
L \frac{\mathrm{d}}{\mathrm{dt}} p_{s r}=-\frac{c^{2}}{f_{0}} q_{*}
$$

где $p_{s r}$ - среднее гидростатическое давление по участку $0 \leq x \leq L$.

Принимая во внимание равенство производных от среднего давления и давления в сечении датчика, приходим к дифференциальному уравнению:

$$
\begin{gathered}
\frac{d p_{d}}{d t}=\mathrm{Y}\left(p_{d}, s_{*}, z_{*}\right)=-\frac{\mu \rho_{0} s_{*} c^{2}}{L \cdot f_{0}} \sqrt{2 g \zeta_{p}\left[z_{d}-z_{*}+\frac{p_{d}-p_{a t m}}{\rho_{0} g}\right]}, \\
\zeta_{p}=1+\frac{1}{K_{g}}\left[p_{d}-p^{\mathrm{O}}+\rho_{0} g\left(z_{d}-z_{*}\right)\right] .
\end{gathered}
$$

Применительно к случаю нескольких утечек (i) аналог (11) можно записать следующим образом:

$$
\begin{gathered}
\frac{d p_{d}}{d t}=\mathrm{Y}\left(p_{d}, s_{*_{1}}, \ldots, s_{*_{i}}, z_{* 1}, \ldots, z_{*_{i}}\right)=-\frac{\mu \rho_{0} c^{2}}{L \cdot f_{0}} \sum_{i} s_{*_{i}} \cdot \sqrt{2 g \zeta_{p i}\left[z_{d}-z_{*_{i}}+\frac{p_{d}-p_{a t m}}{\rho_{0} g}\right]}, \\
\zeta_{p i}=1+\frac{1}{K_{g}}\left[p_{d}-p^{\mathrm{O}}+\rho_{0} g\left(z_{d}-z_{*_{i}}\right)\right] .
\end{gathered}
$$

Если все сечения утечек имеют одну и ту же высотную отметку, то последнее уравнение сводится к (11) при этом $s_{*}=\sum_{i} s_{*_{i}}$. Отсюда следует, что решение задачи восстановления параметров $s_{*}, z_{*}$ в общем случае не дает возможности получить индивидуальные параметры утечки, однако значительно облегчает их поиск. Учитывая это обстоятельство и полагая, что результаты анализа данной задачи будут справедливы и для более 
сложных вариантов утечек, целесообразно остановиться на уравнении (11) более подробно.

Разделяя переменные в уравнении (11) и интегрируя, можно получить в квадратурах интегральную кривую, проходящую через точку $\left.p_{d}\right|_{t=0}=p_{01}$. Для несжимаемой жидкости $\left(\zeta_{p} \equiv 1\right)$ при заданных параметрах $s_{*}=\hat{s}_{*}, z_{*}=\hat{z}_{*}$ интегральная кривая имеет явный вид:

$$
\begin{gathered}
\hat{p}_{d}=\varphi(t)=p_{a t m}+\rho_{0} g \cdot\left(\hat{z}_{*}-z_{d}\right)+\frac{\rho_{0}}{2} \cdot(\hat{A}+\hat{B} t)^{2} \\
\hat{\mathrm{A}}=\sqrt{2 g\left[z_{d}-\hat{z}_{*}+\frac{p_{01}-p_{a t m}}{\rho_{0} g}\right]} ; \hat{\mathrm{B}}=-\frac{\mu \hat{s}_{*} c^{2}}{L f_{0}} .
\end{gathered}
$$

Далее величины, отмеченные верхним диакритическим знаком сิ, отнесены к решению прямой задачи с эталонными значениями $\widehat{\mathrm{S}}_{*}, \widehat{\mathrm{Z}}_{*}$ параметров утечки (в отличие этих же величин при других значениях $\mathbf{s}_{*}, \mathbf{z}_{*}$ ).

Уравнение (12) при заданном давлении $p_{d}^{z m}(t)$ (полученном при опрессовке участка) используют для нахождения параметров $s_{*}, z_{*}$. Соответствующая итерационная процедура с использованием метода наименьших квадратов изложена в [5]. Полученное значение высотной отметки при наложении на профиль участка дает дополнительную (в некоторых случаях исчерпывающую) информацию о наиболее вероятных точках или секторах местонахождения утечки.

\section{Тестовая постановка обратной задачи}

В данной статье расчетные показания датчика $\hat{p}_{d}$ из соотношения (12) использованы в качестве натурных данных (модельного наблюдения). В эти “точные” наблюдения затем вносились ошибки с целью выяснения потенциальных возможностей метода функций чувствительности [6] в установлении параметров утечки. Далее задача формулируется следующим 
образом. Необходимо найти параметры $s_{*}, z_{*}$ из условия минимума функционала (целевой функции).

$$
I=\int_{0}^{\mathrm{T}}\left[\varphi(t)-p\left(x_{d}, t\right)\right]^{2} d t
$$

Условия минимума функционала по параметрам $z_{*}, s_{*}$ после равномерной дискретизации подынтегральной функции в интервале $0 \leq t \leq \mathrm{T}$ имеют вид:

$$
\frac{\partial \mathrm{I}}{\partial z_{*}}=-2 \frac{\mathrm{T}}{\mathrm{N}} \sum_{n=1}^{N}\left(\varphi_{n}-\left.p_{d}\right|_{n}\right)\left(\frac{\partial p_{d}}{\partial z_{*}}\right)_{n}=0, \quad \frac{\partial \mathrm{I}}{\partial s_{*}}=-2 \frac{\mathrm{T}}{\mathrm{N}} \sum_{n=1}^{N}\left(\varphi_{n}-\left.p_{d}\right|_{n}\right)\left(\frac{\partial p_{d}}{\partial s_{*}}\right)_{n}=0
$$

Функции чувствительности [7] в данном случае определяются как

$$
\frac{\partial p_{d}}{\partial s_{*}}=\alpha_{1} ; \quad \frac{\partial p_{d}}{\partial z_{*}}=\alpha_{2}
$$

Дифференциальные уравнения для функций $\alpha_{1}, \alpha_{2}$ получим из (11) дифференцированием по параметру $s_{*}$ и $z_{*}$ соответственно:

$$
\begin{aligned}
& \frac{d \alpha_{1}}{d t}=\frac{\partial \mathrm{Y}}{\partial s_{*}}+\alpha_{1} \frac{\partial \mathrm{Y}}{\partial p_{d}}, \\
& \frac{d \alpha_{2}}{d t}=\frac{\partial \mathrm{Y}}{\partial z_{*}}+\alpha_{2} \frac{\partial \mathrm{Y}}{\partial p_{d}} .
\end{aligned}
$$

С начальным условием, вытекающим из (13) при $\mathrm{t}=0$ :

$$
\alpha_{1}(0)=0 ; \quad \alpha_{2}(0)=0
$$

Уравнения (14), (15) при заданных параметрах $s_{*}$ и $z_{*}$ могут быть решены совместно с уравнением (11). Для несжимаемой жидкости аналитическое решение системы уравнений (14), (15) имеет вид:

$$
\alpha_{1}=\rho_{0} \frac{\mathrm{B}}{S_{*}}(\mathrm{~A}+\mathrm{B} t) t, \quad \alpha_{2}=-\rho_{0} g \frac{\mathrm{B}}{\mathrm{A}} t .
$$

Итерационный процесс по определению $s_{*}$ и $z_{*}$ строится следующим образом. Пусть $s_{*}^{\kappa}$ и $z_{*}^{m}$ некоторые приближенные значения искомых 
параметров. Следуя [4] при фиксированной величине $z_{*}=\bar{z}_{*}$, представим $p_{d}(t)$ с точностью до членов $O\left(\left(s_{*}-s_{*}^{\kappa}\right)^{2}\right)$ в виде:

$$
p_{d}\left(t, s_{*}, \bar{z}_{*}\right) \approx p_{d}\left(t, s_{*}^{K}, \bar{z}_{*}^{m}\right)+\alpha_{1}^{K}\left(t, \bar{z}_{*}^{m}\right)\left(s_{*}-s_{*}^{K}\right)
$$

Введем обозначения

$p_{d}\left(t, s_{*}^{K}, \bar{z}_{*}^{m}\right)=p_{n m}^{K} ; p_{d}\left(t, \bar{s}_{*}^{K}, z_{*}^{m}\right)=p_{n \kappa}^{m} ; \alpha_{1}\left(t, s_{*}^{K}, \bar{z}_{*}^{m}\right)=\alpha_{1 n m}^{\kappa} ; \alpha_{2}\left(t, \bar{s}_{*}^{K}, z_{*}^{m}\right)=\alpha_{2 n \kappa^{*}}^{m}$

С учетом принятых обозначений из необходимого условия минимума функционала (13) путем подстановки (17) и выделения $s_{*}$ получим

$$
s_{*} \approx s_{* m}^{K}+\sum_{n=1}^{N} \alpha_{1 n m}^{K} \cdot\left(\varphi_{n}-p_{n m}^{K}\right) / \sum_{n=1}^{N}\left(\alpha_{1 n m}^{K}\right)^{2}
$$

Формула (18) дает значение $s_{* m}^{\kappa}$ на следующем шаге итерации:

$$
s_{* m}^{K+1}=s_{* m}^{K}+\sum_{n=1}^{N} \alpha_{1 n m}^{K} \cdot\left(\varphi_{n}-p_{n m}^{K}\right) / \sum_{n=1}^{N}\left(\alpha_{1 n m}^{K}\right)^{2}
$$

Аналогичным образом можно получить формулу, дающую значение $z_{* K}^{m+1}$ на следующем шаге итерации

$$
z_{* K}^{m+1}=z_{* K}^{m}+\sum_{n=1}^{N} \alpha_{1 n m}^{\kappa} \cdot\left(\varphi_{n}-p_{n K}^{m}\right) / \sum_{n=1}^{N}\left(\alpha_{1 n m}^{\kappa}\right)^{2}
$$

Излагаемый итерационный процесс (являющийся модификацией метода Ньютона) рассмотрен в работе [4] применительно к задаче утечки при установившемся течении жидкости в трубопроводе. Алгоритм имеет квадратичную сходимость при достаточно хорошем начальном приближении. 


\section{Алгоритмы поиска решения}

Дальнейшая организация итеративного процесса допускает различные варианты, эффективность которых может быть оценена путем численных параметрических расчетов:

a) высотный профиль контролируемого участка трубопровода известен:

- в качестве начального приближения параметры утечки задают следующим образом: $0<s_{*}<10^{-9} \mathrm{M}^{2}, z_{*}=z_{\max }$;

- согласно формуле (19) получают следующее приближение $s_{* m}^{\kappa+1}$;

- при фиксированном значении параметра $z_{* \kappa}^{m} \quad$ продолжают итерации до выполнения условия $\left|s_{* m}^{\bar{\kappa}+1}-s_{* m}^{\bar{\kappa}}\right| \leq \varepsilon_{s} \quad$ (на итерации $\kappa=\bar{\kappa})$;

- при фиксированном значении параметра $s_{* m}^{\bar{\kappa}}$ осуществляют итерации по формуле (20) до выполнения условия $\left|z_{* \bar{k}}^{\bar{m}+1}-z_{* \bar{k}}^{\bar{m}}\right| \leq \varepsilon_{z}$ (на итерации $m=\bar{m}$ );

- величину $z_{* \bar{K}}^{\bar{m}}$ сравнивают $\mathrm{c}$ исходным значением $z_{* \kappa}^{m}$. Если выполнено условие $\left|z_{* \bar{K}}^{\bar{m}}-z_{* K}^{m}\right| \leq \varepsilon_{z}$, итеративный процесс считают завершенным;

- иначе полагают $s_{* m}^{K}=s_{* m}^{\bar{K}} z_{* \kappa}^{m}=z_{* \bar{K}}^{\bar{m}}$ и повторяют итерационную процедуру, начиная с формулы (19).

b) начальное приближение задают идентично предыдущему варианту:

- согласно формуле (19) получают следующее приближение $s_{* m}^{\kappa+1}$;

- согласно формуле (20) получают приближение $z_{*_{k}+1}^{m+1}$;

- по формуле (19) находят приближение $s_{* m+1}^{\kappa+1}$;

- проверяют выполнение условий завершения итерационного процесса:

$$
\left|s_{* m+1}^{\kappa+1}-s_{* m}^{\kappa}\right| \leq \varepsilon_{S} \text { И }\left|z_{* K+1}^{m+1}-z_{* K}^{m}\right| \leq \varepsilon_{z}
$$


- если условия (21) не выполнены, то итерационный цикл повторяют.

c) в отличие от варианта $a$ ) меняют местами параметры внутреннего $s_{*}$ и внешнего $z_{*}$ цикла.

Оценку перечисленных вариантов итерационной процедуры будем проводить с учетом того, что функции $\varphi, p_{d}, \alpha_{1}, \alpha_{2}$ определены аналитически. Эти обстоятельства позволяют, при необходимости, использовать вместо сумм конкретизированное аналитическое представление соответствующих интегралов, что эквивалентно неограниченному увеличению количества замеров.

Условия сходимости итераций (19), (20) (условия минимума функционала (13)) после преобразований могут быть записаны в виде следующих двух алгебраических уравнений, относительно $S_{*}, z_{*}$ :

$$
\hat{\mathrm{A}} \hat{\mathrm{B}}-\mathrm{AB}=0, \quad \hat{\mathrm{B}}^{2}-\mathrm{B}^{2}=0 .
$$

Из (22) следует

$$
z_{*}=\hat{z}_{*}, \quad S_{*}=\hat{s}_{*} .
$$

Метод функций чувствительности при условии сходимости итерационной процедуры и полном отсутствии различного рода ошибок (неадекватности математической модели, погрешности в исходной информации, ошибки аппроксимации исходной задачи, ошибки округления при выполнении вычислительных операций и т.д.) позволяет получить точное решение задачи.

\section{Неограниченное число измерений. Влияние возмущающих факторов}

Решение обратной задачи, как правило, чувствительно к вышеупомянутым ошибкам. При этом бывает трудно выяснить, каким конкретно фактором вызвано отклонение результата численного расчета от 
реального измерения. Представляет интерес выявление степени влияния этих факторов на суммарную погрешность «восстановленного» решения. В связи с этим целесообразна оценка влияния систематической и инструментальной погрешности измерения. Предположим, что все замеры давления лежат ниже расчетных значений, но в пределах погрешности измерения. Такой вариант соответствует наличию некоторой неучтенной систематической ошибки $\varepsilon_{\delta}$, и «кривая замеров» может быть представлена следующим соотношением:

$$
\tilde{p}_{d}=\hat{p}_{d}-\varepsilon_{\delta}
$$

где $\tilde{p}_{d}-$ точное решение адекватной (идентифицированной) модели $\hat{p}_{d}$, заданное с ошибкой $\varepsilon_{\delta}$.

Погрешность восстановления параметров утечки в этом случае может быть определена явным образом и имеет вид:

$$
\begin{gathered}
\varepsilon_{S_{*}}=\sqrt{1+\frac{20}{3 \mathrm{~T}^{2}} \frac{L^{2} f_{0}^{2}}{\rho_{0} \mu^{2} c^{4} \hat{\mathrm{s}}_{*}^{2}} \varepsilon_{\delta}}-1, \\
\varepsilon_{z_{*}}=\frac{2}{g \hat{z}_{*}} \frac{5 \widehat{\mathrm{A}}^{2}-6 \widehat{\mathrm{A}} \widehat{\mathrm{B}} \mathrm{T}+12 \frac{\varepsilon_{\delta}}{\rho_{0}}}{3 \rho_{0} \widehat{\mathrm{B}}^{2} \mathrm{~T}^{2}+20 \varepsilon_{\delta}} \varepsilon_{\delta} .
\end{gathered}
$$

Рисунок 1 иллюстрирует влияние систематической ошибки на точность восстановления параметров утечки методом функций чувствительности в зависимости от величины временного интервала, на котором осуществлялась идентификация. Исходные данные для проведения расчетов выбраны следующим образом: $L=3 \cdot 10^{4} \mathrm{~m} ; D=1 \mathrm{M} ; p_{01}=3 \cdot 10^{6}$ Па ; $\rho_{0}=850 \mathrm{\kappa} \Gamma / \mathrm{M}^{3} ; \quad \mathrm{c}=1000 \mathrm{M} / \mathrm{c} ; \quad \mu=0.5 ; \quad \mathrm{z}_{d}=100 \mathrm{M} ; \quad \hat{\mathrm{z}}_{*}=80 \mathrm{M} ; \quad \hat{\mathrm{S}}_{*}=2 \cdot 10^{-4} \mathrm{M}^{2} ;$ $\varepsilon_{\delta}=3 \cdot 10^{3} \Pi \mathrm{a}$ 


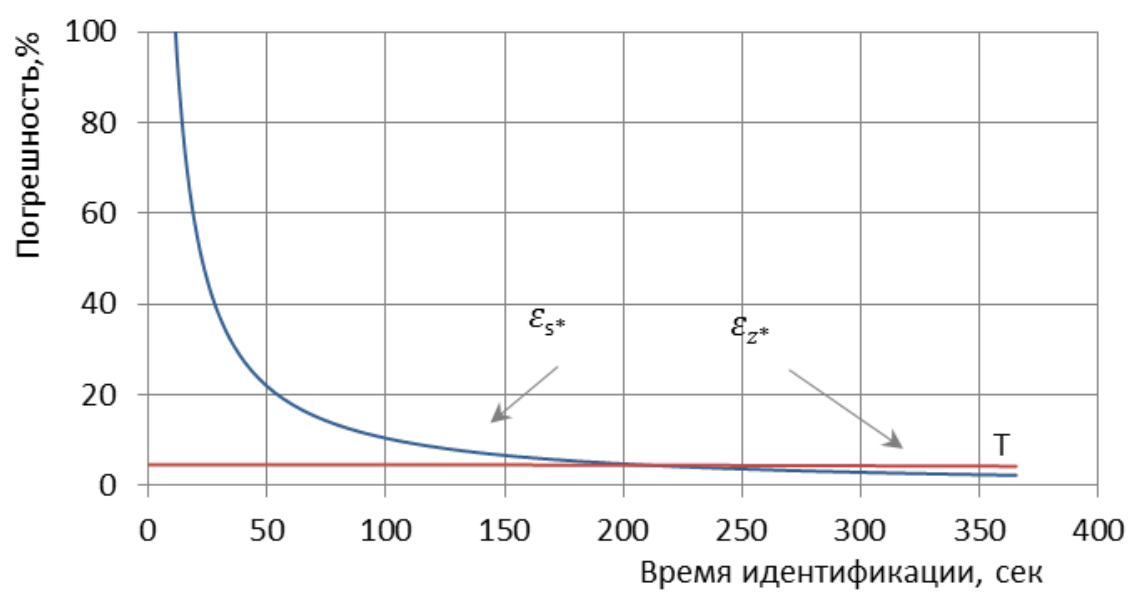

Рисунок 1. Зависимость погрешности восстановления параметров утечки от интервала

Из рисунка 1 следует, что сходимость к эталонному решению по параметру $z_{*}$ требует более длительных интервалов идентификации и более жестких требований к величине $\varepsilon_{z_{*}}$ критерия сходимости итераций. Параметрические расчеты показали, что при решении обратной задачи в рамках метода функций чувствительности целесообразно ставить дополнительное требование на минимальную величину временного интервала, начиная с которого возможно гарантированное осуществление идентификации в условиях неограниченного числа замеров. Это требование можно интерпретировать как минимальное необходимое время определения параметров утечки с помощью метода функций чувствительности (предварительный интервал накопления информации). Для данного варианта это время составляет величину порядка 25 с (0,13\% от общего времени истечения, при этом вытекает 0,26 \% от предельного объема утечки). Величину падения давления на предварительном интервале $\quad \Delta p_{\Lambda}=p_{01}-\hat{p}_{d 1}$ будем трактовать как минимальную амплитуду волны разрежения, которая может быть идентифицирована методом функций чувствительности в нестационарном процессе при 
наличии возмущений и ошибок измерения. В данном варианте величина $\Delta p_{\Lambda}$ составляет 7766 Па.

Аналогичным образом можно оценить влияние периодических возмущений в замерах давления, придавая «точным замерам» $\hat{p}_{d}$ синусоидальное возмущение с амплитудой в пределах погрешности измерения реального датчика. Воздействие полигармонических сигналов в широком диапазоне изменения частот заменим последовательным применением моногармонических воздействий с разными частотами и рассмотрим реакцию на них:

$$
\tilde{p}_{d}=\hat{p}_{d}+\varepsilon_{\delta} \sin \omega t
$$

после преобразований, аналогичных выводу (22), в итоге получим:

$$
\begin{gathered}
s_{*}^{2}=\hat{s}_{*}^{2}+\frac{4}{\rho_{0} \mathrm{~T}^{4}}\left(\frac{L f_{0}}{\mu c^{2}}\right)^{2}\left[\frac{5}{\omega^{2}} \sin \omega \mathrm{T}-\frac{\mathrm{T}}{\omega} \cos \omega \mathrm{T}\right] \cdot \varepsilon_{\delta}, \\
z_{*}=z_{d}+\frac{1}{\rho_{0} g}\left(p_{01}-p_{a t m}\right)-\frac{1}{2 g}\left\{\hat{\mathrm{A}} \frac{\hat{\mathrm{B}}}{\mathrm{B}}+\frac{12}{\rho_{0} \mathrm{BT}}\left[\frac{\mathrm{T}}{\omega} \cos \omega \mathrm{T}-\frac{6}{\omega^{2}} \sin \omega \mathrm{T}\right] \cdot \varepsilon_{\delta}\right\}^{2} .
\end{gathered}
$$

Опуская слагаемые с порядком малости выше первого, запишем (24) в виде:

$$
\begin{gathered}
s_{*}=\hat{s}_{*}+\frac{2}{\rho_{0} \hat{s}_{*} \mathrm{~T}^{4}}\left(\frac{L f_{0}}{\mu c^{2}}\right)^{2}\left[\frac{5}{\omega^{2}} \sin \omega \mathrm{T}-\frac{\mathrm{T}}{\omega} \cos \omega \mathrm{T}\right] \cdot \varepsilon_{\delta} \\
z_{*}=\hat{z}_{*}-\frac{2 \hat{\mathrm{A}}}{\rho_{0} g}\left\{\left[\frac{6}{\hat{\mathrm{B} \mathrm{T}^{2}}}+\frac{\hat{\mathrm{A}}}{\hat{s}_{*}^{2} \mathrm{~T}^{3}}\left(\frac{L f_{0}}{\mu c^{2}}\right)^{2}\right] \frac{\cos \omega \mathrm{T}}{\omega}-\left[\frac{36}{\hat{\mathrm{BT}}^{3}}+\frac{5 \hat{\mathrm{A}}}{\hat{s}_{*}^{2} \mathrm{~T}^{4}}\left(\frac{L f_{0}}{\mu c^{2}}\right)^{2}\right] \frac{\sin \omega \mathrm{T}}{\omega^{2}}\right\} \cdot \varepsilon_{\delta} .
\end{gathered}
$$

На рисунке 2 представлены результат учета периодических возмущений с частотой 7 Гц в «кривых замера» давления (шум измерения) и их влияние на точность определения параметров утечки в зависимости от протяженности временного интервала, на котором осуществлена идентификация. Представленный результат получен при наборе исходных данных, использованном ранее при анализе систематической ошибки 
(рисунок 1). Минимальная необходимая величина временного интервала при этом увеличилась втрое, а величина $\Delta p_{\Lambda}$ составила 22940 Па при амплитуде периодического возмущения 3·103 Па. При частоте 0,7 Гц эти величины составляют 3 мин и 51874 Па соответственно.

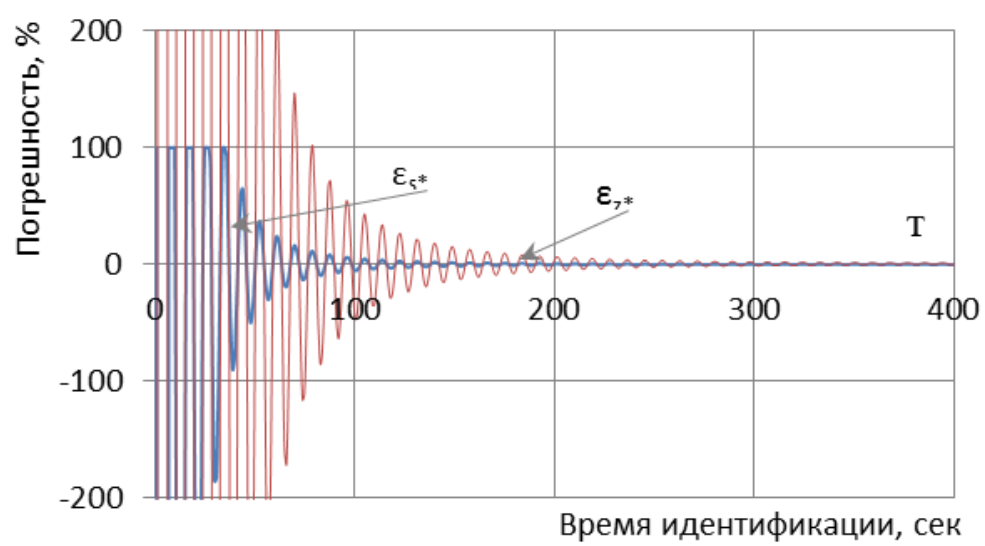

Рисунок 2. Влияние периодического возмущения частотой 7 Гц на погрешность идентификации параметров утечки

\section{Обсуждение результатов}

Процесс восстановления носит колебательный характер. Наблюдается некоторая аналогия с работой [8], где показано как увеличение количества замеров по времени ведет к повышению чувствительности и колебательному процессу восстановления параметра в математической модели нестационарной фильтрации жидкости в пористой среде.

Установлено, что оптимальная для измерений давления точка контролируемого участка находится в сечении с минимальной отметкой высотного профиля. Минимальный необходимый предварительный интервал идентификации наиболее существенным образом зависит от размера отверстия утечки.

Последнее обстоятельство иллюстрирует рисунок 3, на котором в зависимости от длительности используемого интервала данных по измерению давления представлено влияние на погрешность определения 
параметров утечки с $\hat{s}_{*}=2 \cdot 10^{-5}$ периодических возмущений с частотой 0,7 Гц.

Предварительный интервал в этом варианте составляет величину свыше 13 мин и $\Delta p_{\Lambda}-$ не менее $2.4 \cdot 10^{4}$ Па.

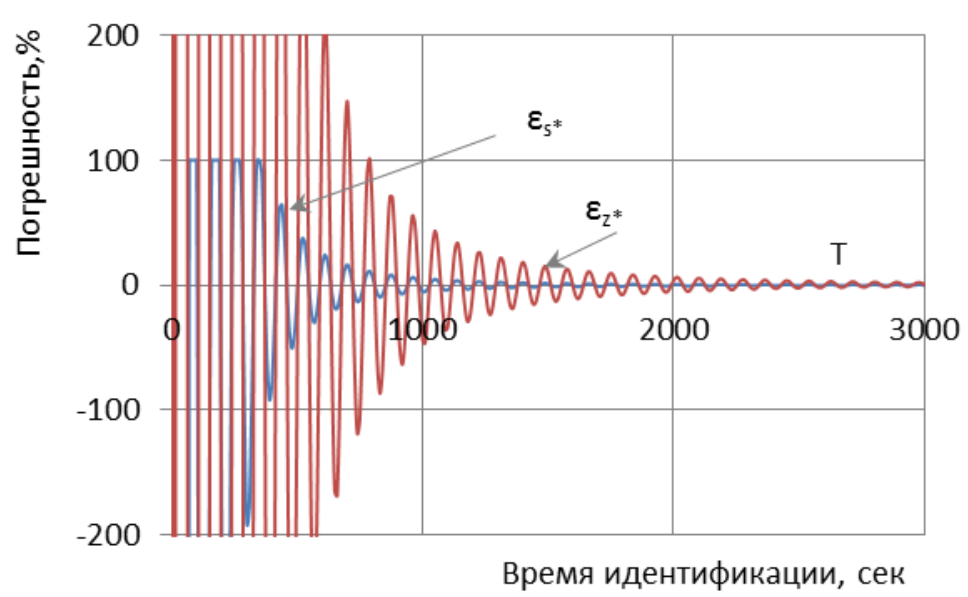

Рисунок 3. Влияние периодического возмущения частотой 0,7 Гц на погрешность идентификации параметров утечки

Для выделения полезного сигнала часто применяют различные способы сглаживания по одной реализации переходного процесса [9]. Процедура сглаживания измеренных временных рядов (фильтрация ошибок измерения), применяемая в предварительном интервале $0 \leq t \leq \mathrm{T}_{\kappa}$, по формуле:

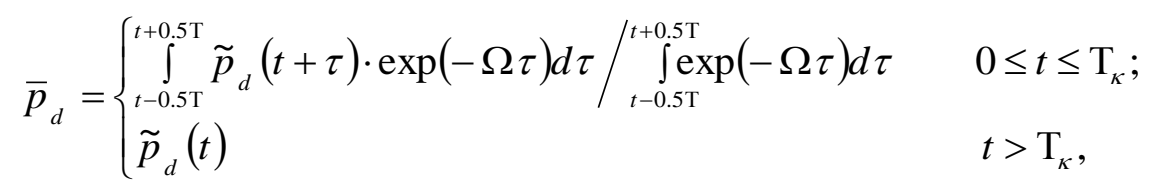

или сглаживание экспериментальных данных на основе скользящего усреднения на интервале т в окрестности текущего значения времени $t$ по формуле

$$
\bar{p}_{d}=\frac{1}{\mathrm{~T}} \cdot \int_{t-0.5 \mathrm{~T}}^{t+0.5 \mathrm{~T}} \tilde{p}_{d}(\tau) d \tau
$$


позволяют расширить границы применимости рассматриваемого метода функций чувствительности (в части сужения необходимого интервала измерений и снижения амплитуды идентифицируемой волны). Влияние неизбежного смещения оценки $\bar{p}_{d}(t)$ при процедурах сглаживания (26), (27), соответствующего прохождению зашумленного сигнала через некоторый фильтр, отделяющий низкочастотный полезный сигнал от более высокочастотной помехи, устраняется в процессе идентификации. Следует отметить, что учет априорной информации об искомом решении, например, об интервале изменения высотных отметок контролируемого участка, позволяет значительно ускорить сходимость. Последнее обстоятельство иллюстрирует рисунок 4, на котором представлен результат расчета при исходных данных соответствующих рисункам 2, 3, но с учетом того, что высотные отметки контролируемого участка находятся в пределах $10 \div 125$ м. Эта информация позволяет снижать амплитуду «шума расчетной модели», запрещая выброс значений $z_{*}^{m+1}$ за пределы данного интервала. Реализация ограничения величины $z$ заданным интервалом аналогична введению «штрафа» за большое отклонение нового значения $z_{*}^{m+1}$ от значения $z_{*}^{m}$ на предыдущей итерации. Величина временного интервала идентификации варьировалась в пределах указанных на рисунке 4.
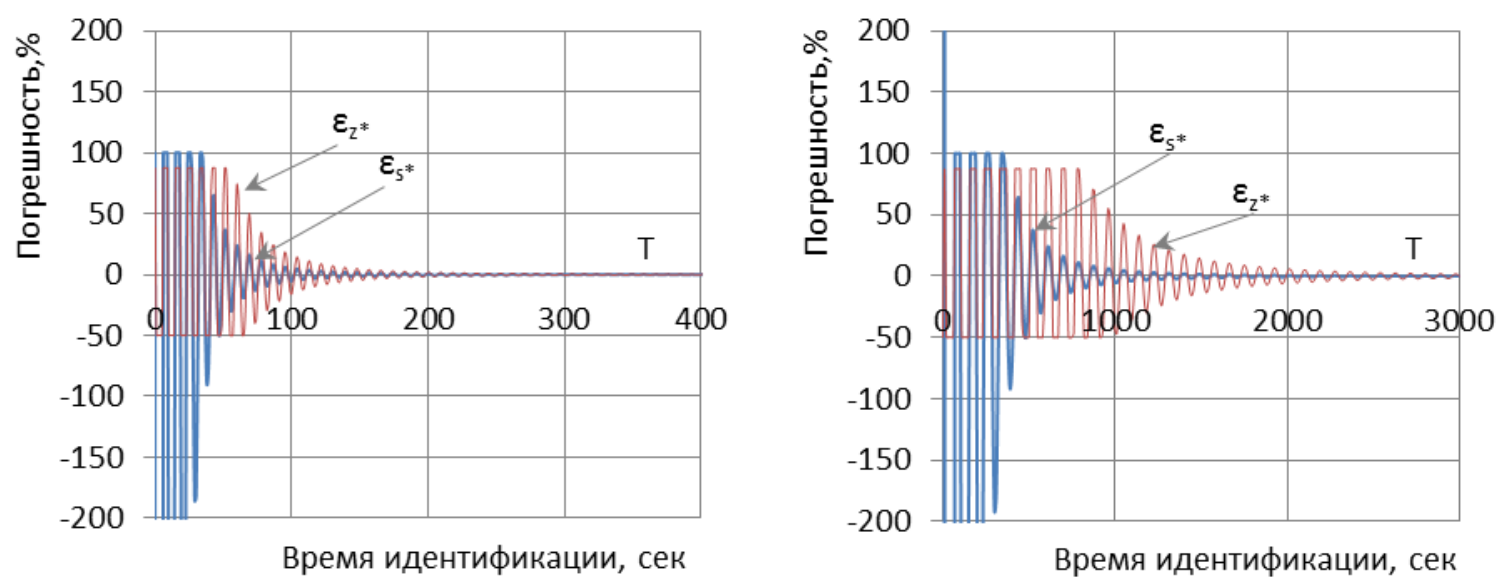

Рисунок 4. Влияние частоты возмущения на погрешность восстановления параметров утечки 
Как показали вычисления, введение процедуры «штрафа» эффективно в случае высокочастотных возмущений и практически не проявляется для низких частот.

Следует отметить, что минимальное значение идентифицируемой площади отверстия при параметрических расчетах составляло $2 \cdot 10^{-8} \mathrm{~m}^{2}$. Для идентификации параметров утечки в этом варианте потребовалась информация о протекании процесса на временном интервале более 2 сут, $15 \%$ которого составлял предварительный интервал. Применение исходных данных с бесконечным числом точек «точного» наблюдения гарантирует сходимость к эталонному решению (или его разностному аналогу). Совпадение решения обратной задачи с эталонными значениями $\hat{s}_{*}$ и $\hat{z}_{*}$ имеет место только в модельных ситуациях, когда исходные данные фактически не содержат ошибок, поскольку они заданы аналитическим решением (как в данном случае) или вычислены на ЭВМ путем решения прямых задач.

\section{Дискретные измерения}

При дискретном задании функции $\varphi$ помимо выбора варианта итеративной процедуры и интервала идентификации возникает вопрос выбора величины дискреты (количества замеров). Для выяснения этих вопросов проведены расчеты по трем вариантам организации итеративного процесса с различными значениями т и $N$. Установлено, что варианты $a), b), c)$ неэффективны. Вероятной причиной является наличие большого числа локальных минимумов целевой функции. Даже при задании начального приближения $s_{*}^{0}$, в точности равного $\hat{s}_{*}$ сходимость к точному решению не обеспечивается. В то же время начальное приближение высотной отметки утечки $z_{*}^{0}$, в точности равное $\hat{z}_{*}$ (в отсутствии «зашумленности»), обеспечивает быструю сходимость при произвольном варьировании числа дискретных моментов времени. Эти обстоятельства 
свидетельствуют о необходимости и возможности разработки дополнительной процедуры поиска глобального минимума. Для ее построения вместо итерационной формулы (20) используем процедуру перебора значений $z_{*}$ в интервале фактического перепада $\left(z_{\min } \leq z_{*} \leq z_{\max }\right)$ на контролируемом участке, которую можно организовать следующим образом $[6$, с. 81$]$ :

$$
z_{*}^{m+1}=z_{*}^{m}-\Delta z ; \quad z_{*}^{0}=z_{\max }, \quad \Delta z=\left(z_{\max }-z_{\min }\right) / n
$$

Для каждого значения $z_{*}^{m}$ с помощью итераций по формуле (19) вычисляем соответствующую величину ${ }^{{ }_{*}{ }^{\text {mm }}}$ и величину целевой функции. По завершении перебора принимаем параметры $s_{* *}^{\kappa m}, z_{* *}^{m}$, относящиеся к минимальной величине в ряду значений целевой функции, за приближенную оценку искомого решения. Уточнение проводим путем уменьшения шага $\Delta z$ и повторения процедуры перебора, начиная с точки $z_{* * *}^{m}$ или её аналога в последующих уточнениях. Итерации считаем завершенными по достижении шага $\Delta z$ величины 10-2 м. За начальное приближение площади отверстия целесообразно принимать $s_{*}^{0}<10^{-8} \mathrm{M}^{2}$.

В качестве иллюстрации вышесказанного на рисунке 5 представлены кривые, отражающие процесс итерационного приближения к точному решению задачи (глобальному минимуму целевой функции). Как видно из рисунка 5, предлагаемый алгоритм обеспечивает (при отсутствии возмущений) сходимость к точному решению.

Однако он достаточно трудоемок и высока вероятность получения эталонного решения лишь за 20-30 итераций. Кроме этого сходимость к точному решению, как показано далее, наблюдается лишь в том случае, когда минимальная величина в ряду значений целевой функции находится внутри расчетной области. Расположение минимальной величины на границе области расчета, как правило, соответствует сходимости к параметрам, отличающимся от эталонных параметров. 

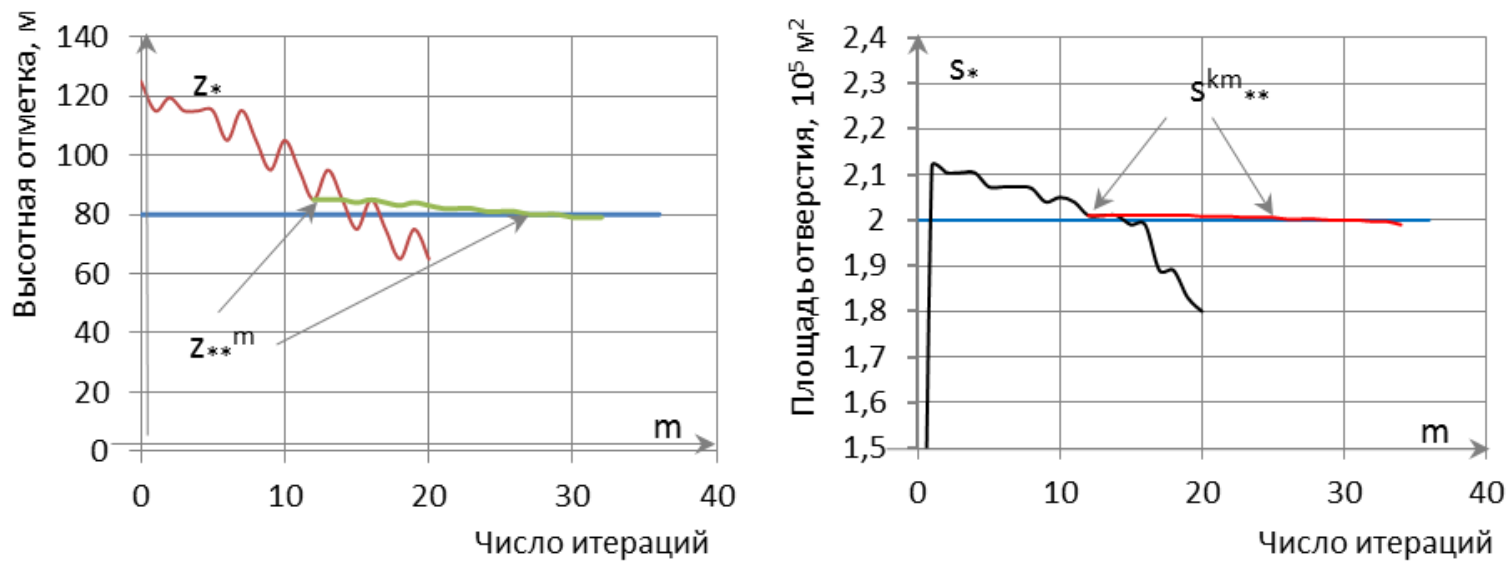

Рисунок 5. Сходимость определяемых параметров

Локальные минимумы на представленных кривых зависимости параметров утечки $z_{*}^{m}, s_{*}{ }^{\text {km }}$ от числа итераций $m$ соответствуют локальным минимумам целевой функции. Попадание в локальный минимум приводит к сходимости итераций, причем изменение начального приближения не гарантирует (без применения специальных мер) выхода на глобальный минимум целевой функции. Следует отметить, что отклонение численного решения от натурных данных может быть неправомерно отнесено к погрешности параметрического метода, а не к алгоритму получения решения. Некоторые алгоритмы могут давать неприемлемый результат даже при абсолютно адекватной детерминированной математической модели. Это обстоятельство следует учитывать при анализе погрешности параметрических методов обнаружения утечек, в которых используют итерационные процессы.

Эффективность предлагаемого алгоритма рассмотрена в условиях периодических возмущений при варьировании числа замеров и величины временного интервала идентификации. Для этого случая процесс схождения к эталонному решению существенным образом зависит от числа точек наблюдения (помимо интервала идентификации, амплитуды и частоты возмущений). Трудоемкость итерационного процесса приблизительно такая же, как и в предыдущем случае. Это обстоятельство 
демонстрирует рисунок 6, на котором представлено количество итераций, требуемых для сходимости.
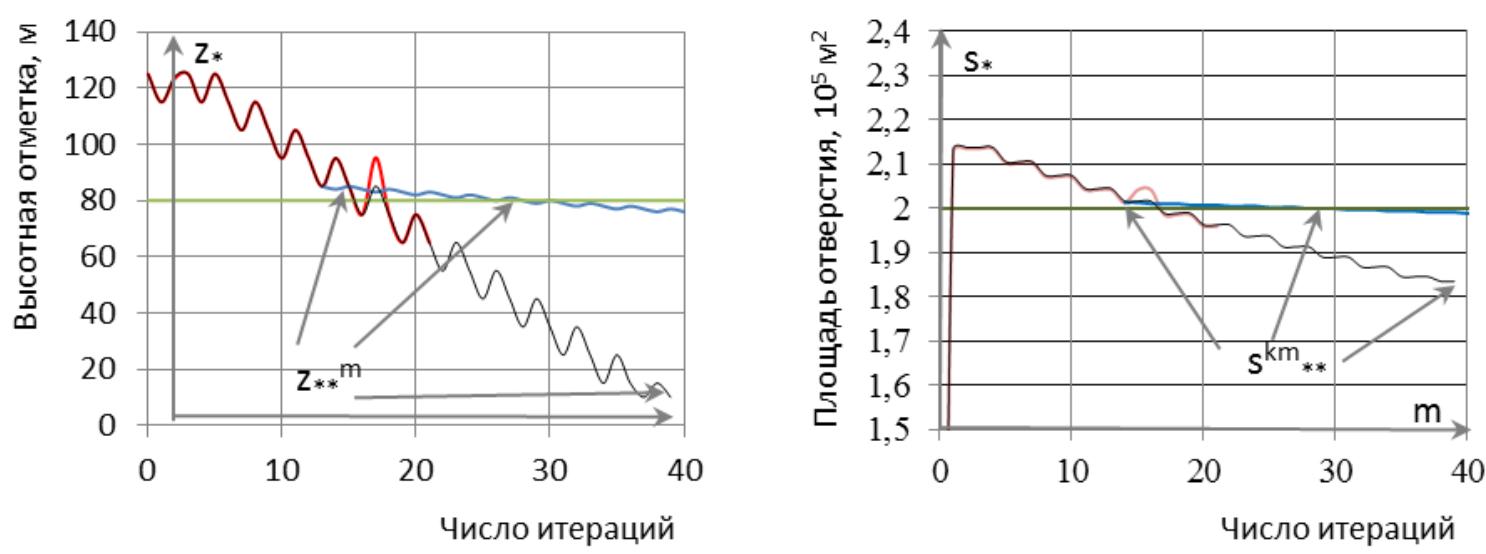

Рисунок 6. Сходимость по известному и предлагаемому алгоритмам

Как ни странно, лучший результат соответствует более грубой временной сетке, которая дает сходимость к точному решению. На более мелкой сетке наблюдается сходимость к решению, существенно отличающемуся от эталонного решения. Таким образом, выбор шага временной дискретизации может оказаться в числе решающих факторов успешной реализации метода.

\section{Альтернативные тесты}

Данное обстоятельство может формулироваться следующим образом: существуют возмущения, которые максимально затрудняют идентификацию параметров утечки. В связи с этим возникает задача выявления места нахождения источников возмущений и нейтрализации их влияния. Тестирование соответствующих алгоритмов предпочтительно проводить на модификациях рассмотренного выше элементарного решения.

Рассмотрим модификации точного решения в части учета зависимости от времени площади отверстия утечки и противодавления внешней среды, учета влияния теплообмена и акустических возмущений со стороны 
внешней среды (в том числе искусственного происхождения), приводящие к явным аналитическим зависимостям $p_{d}(t)$.

\section{Две утечки с различными моментами возникновения}

Аналог уравнения (11) принимает вид:

$$
\frac{d p_{d}}{d t}=\left\{\begin{array}{cc}
Y_{1}=-\frac{\mu \rho_{0} c^{2}}{L f_{0}} s_{* 1} \cdot \sqrt{2 g \zeta_{p 1}\left[z_{d}-z_{* 1}+\frac{p_{d 1}-p_{a t m}}{\rho_{0} g}\right]} & 0 \leq t<\Delta \tau \\
Y_{2}=-\frac{\mu \rho_{0} c^{2}}{L f_{0}} \sum_{i=1}^{2} s_{* i} \cdot \sqrt{2 g \zeta_{p i}\left[z_{d}-z_{* i}+\frac{p_{d 2}-p_{a t m}}{\rho_{0} g}\right]} & \Delta \tau<t \leq \mathrm{T} \\
\zeta_{p i}=1+\frac{1}{\mathrm{~K}_{g}}\left[p_{d}-p^{o}+\rho_{0} g\left(z_{d}-z_{* i}\right)\right] .
\end{array}\right.
$$

Решение уравнения (28) в первом временном диапазоне $0 \leq t<\Delta \tau$ при $\zeta_{p i}=1$ рассмотрено ранее. Интегральную кривую, проходящую через точку $\left.p_{d 1}\right|_{t=0}=p_{10}$, представим следующим образом:

$$
\begin{gathered}
y-y_{0}=-\frac{\mu s_{* 1} c^{2}}{L f_{0}} \cdot t=\mathrm{B} \cdot \mathrm{t} \\
\hat{p}_{d 1}=\varphi_{1}=p_{a t m}+\rho_{0} g\left(\hat{z}_{* 1}-z_{d}\right)+\frac{\rho_{0}}{2}(\widehat{\mathrm{A}}+\widehat{\mathrm{Bt}})^{2} \\
y=\sqrt{2 g\left[z_{d}-z_{* 1}+\frac{p_{d 1}-p_{a t m}}{\rho_{0} g}\right]} ; \quad y_{0}=\sqrt{2 g\left[z_{d}-z_{* 1}+\frac{p_{10}-p_{a t m}}{\rho_{0} g}\right]}=\mathrm{A} .
\end{gathered}
$$

Во втором диапазоне аналитическое решение может быть записано с помощью квадратуры, легко раскрывающейся для варианта $S_{* 1}=s_{* 2}=s_{*}$. Ограничиваясь этим случаем, запишем частное решение (28) в виде:

$$
y_{1}^{3}-y_{2}^{3}=\mathcal{C}+6 g\left(z_{* 2}-z_{* 1}\right) \mathrm{B} \cdot t
$$


здесь $y_{1}=y\left(z_{* 1}, p_{d 2}\right)$ - обозначение принадлежности введенной ранее функции у ко второму диапазону;

$y_{2}=y\left(z_{* 2}, p_{d 2}\right)-$ аналог функции $y_{1}$, относящийся ко второй утечке;

$\mathcal{C}$ - произвольная константа, подлежащая определению из условия равенства давлений на границе диапазонов $\left.p_{d 2}\right|_{t=\Delta \tau}=\left.p_{d 1}\right|_{t=\Delta \tau}=p_{\tau}$.

Согласно (29) интегральную кривую, проходящую через точку $\left.p_{d 2}\right|_{t=\Delta \tau}=p_{\tau}$, для несжимаемой жидкости $\left(\zeta_{p} \equiv 1\right)$ при заданных параметрах $s_{*}^{2}=\hat{s}_{*}, z_{* 1}=\hat{z}_{* 1}, Z_{* 2}=\hat{z}_{* 2}, \Delta \tau=\Delta \hat{\tau}$ можно записать в форме:

$$
\begin{gathered}
\hat{y}_{1}^{3}-\hat{y}_{2}^{3}-\hat{y}_{1 \tau}^{3}+\hat{y}_{2 \tau}^{3}=6 g\left(\hat{z}_{* 2}-\hat{z}_{* 1}\right) \widehat{\mathrm{B}} \cdot(t-\Delta \hat{\tau}), \\
\hat{y}_{1}^{2}-\hat{y}_{2}^{2}=2 g\left(\hat{z}_{* 2}-\hat{z}_{* 1}\right) \\
p_{\tau}=p_{a t m}+\rho_{0} g\left(\hat{z}_{* 1}-z_{d}\right)+\frac{\rho_{0}}{2}(\widehat{\mathrm{A}}+\widehat{\mathrm{B}} \Delta \hat{\tau})^{2} \\
\hat{y}_{1 \tau}= \pm(\hat{A}+\hat{B} \cdot \Delta \hat{\tau}), \quad \hat{y}_{2 \tau}=\sqrt{2 g\left(\hat{z}_{* 2}-\hat{z}_{* 1}\right)+(\hat{A}+\hat{B} \cdot \Delta \hat{\tau})^{2}} .
\end{gathered}
$$

Выражая $\hat{y}_{1}$ из второго уравнения (30), после подстановки в первое уравнение получим алгебраическое уравнение четвертого порядка относительно $\hat{y}_{2}$ :

$$
\begin{gathered}
6 g\left(\hat{z}_{* 2}-\hat{z}_{* 1}\right) \hat{y}_{2}^{4}-2 \mathcal{D} \hat{y}_{2}^{3}+12 g^{2}\left(\hat{z}_{* 2}-\hat{z}_{* 1}\right)^{2} \hat{y}_{2}^{2}+8 g^{3}\left(\hat{z}_{* 2}-\hat{z}_{* 1}\right)^{3}-\mathcal{D}^{2}=0 \\
\mathcal{D}= \pm(\hat{A}+\hat{B} \Delta \hat{\tau})^{3}-2 g\left(\hat{z}_{* 2}-\hat{z}_{* 1}\right)+(\hat{A}+\hat{B} \Delta \hat{\tau})^{3 / 2}+6 g\left(\hat{z}_{* 2}-\hat{z}_{* 1}\right) \hat{B}(t-\Delta \hat{\tau}) .
\end{gathered}
$$

Решение последнего уравнения может быть получено в явном виде, что позволяет также в явном виде записать давление во втором диапазоне как функцию времени: 


$$
\hat{p}_{d 2}=\varphi_{2}=p_{a t m}+\rho_{0} g\left(\hat{z}_{* 2}-z_{d}\right)+\frac{\rho_{0}}{2} \hat{y}_{2}^{2} .
$$

Далее задача формулируется следующим образом. Необходимо в рамках модели (28) по измерениям давления найти параметры $s_{*}, Z_{* 1}, Z_{* 2}, \Delta \tau$ из условия минимума целевой функции. Измерения при тестировании алгоритмов поиска для данного варианта моделируются на основе комбинации точных аналитических решений (формулы (12), (30)). Необходимые условия минимума целевой функции аналогичны (13).

Функции чувствительности в данном случае определяются как

$$
\frac{\partial p_{d i}}{\partial s_{*}}=\alpha_{1 i} ; \frac{\partial p_{d i}}{\partial z_{* 1}}=\alpha_{2 i} ; \frac{\partial p_{d}}{\partial z_{* 2}}=\alpha_{3} ; \frac{\partial p_{d 2}}{\partial(\Delta \tau)}=\alpha_{4}
$$

Дифференциальные уравнения для функций чувствительности получим из (28) по аналогии с (14), (15):

$$
\begin{gathered}
\frac{d \alpha_{1}}{d t}=\left\{\begin{array}{l}
\frac{\partial Y_{1}}{\partial s_{*}}+\alpha_{11} \frac{\partial Y_{1}}{\partial p_{d 1}} \quad 0 \leq t<\Delta \tau \\
\frac{\partial Y_{2}}{\partial s_{*}}+\alpha_{12} \frac{\partial Y_{2}}{\partial p_{d 2}} \quad \Delta \tau<t \leq \mathrm{T}
\end{array} ;\right. \\
\frac{d \alpha_{2}}{d t}=\left\{\begin{array}{l}
\frac{\partial Y_{1}}{\partial z_{* 1}}+\alpha_{21} \frac{\partial Y_{1}}{\partial p_{d 1}} \quad 0 \leq t<\Delta \tau \\
\frac{\partial Y_{2}}{\partial z_{* 1}}+\alpha_{22} \frac{\partial Y_{2}}{\partial p_{d 2}} \quad \Delta \tau<t \leq \mathrm{T}
\end{array} ;\right. \\
\frac{d \alpha_{3}}{d t}=\frac{\partial Y_{2}}{\partial z_{* 2}}+\alpha_{3} \frac{\partial Y_{2}}{\partial p_{d 2}} ; \quad \frac{d \alpha_{4}}{d t}=\alpha_{4} \frac{\partial Y_{2}}{\partial p_{d 2}} ; \quad 0 \leq t<\Delta \tau .
\end{gathered}
$$

Начальные условия имеют вид:

$$
\begin{gathered}
\alpha_{11}(0)=0 ; \quad \alpha_{21}(0)=0 ; \quad \alpha_{12}(\Delta \tau)=\alpha_{11}(\Delta \tau) ; \quad \alpha_{22}(\Delta \tau)=\alpha_{21}(\Delta \tau) ; \\
\alpha_{3}(\Delta \tau)=0 ; \quad \alpha_{4}(\Delta \tau)=\left.\frac{d p_{d 1}}{d t}\right|_{t=\Delta \tau} .
\end{gathered}
$$


Уравнения (32)-(34) при заданных параметрах $\hat{s}_{*}, \hat{z}_{* 1}, \hat{z}_{* 2}, \Delta \hat{\tau}$, могут быть решены совместно с уравнением (28).

Для несжимаемой жидкости эта система уравнений может быть записана в следующей форме:

$$
\begin{gathered}
\frac{d}{d t} \alpha_{11}-\frac{\mathrm{B}}{\mathrm{A}+\mathrm{B} t} \alpha_{11}=\frac{\mathrm{B}}{s_{*}}(\mathrm{~A}+\mathrm{B} t) ; \\
\frac{d}{d t} \alpha_{21}-\frac{\mathrm{B}}{\mathrm{A}+\mathrm{B} t} \alpha_{21}=-\frac{\rho_{0} \mathrm{~g} \mathrm{~B}}{(\mathrm{~A}+\mathrm{B} t)} ; \\
\frac{d}{d y_{2}} \alpha_{12}+\frac{\alpha_{12}}{\sqrt{y_{2}^{2}+2 g\left(z_{* 2}-z_{* 1}\right)}}=\frac{\rho_{0}}{s_{*}} y_{2} ; \\
\frac{d}{d y_{2}} \alpha_{22}-\frac{\alpha_{22}}{\sqrt{y_{2}^{2}+2 g\left(z_{* 2}-z_{* 1}\right)}}=-\frac{\rho_{0}}{2\left(z_{* 2}-z_{* 1}\right)}\left[y_{2}-\frac{y_{2}^{2}}{\sqrt{y_{2}^{2}+2 g\left(z_{* 2}-z_{* 1}\right)}}\right] ; \\
\frac{\alpha_{3}}{d y_{2}} \alpha_{3}+\frac{\rho_{0}}{\sqrt{y_{2}^{2}+2 g\left(z_{* 2}-z_{* 1}\right)}\left[\sqrt{y_{2}^{2}+2 g\left(z_{* 2}-z_{* 1}\right)}-y_{2}\right] ;} \\
\frac{d}{d y_{2}} \alpha_{4}-\frac{\alpha_{4}}{\sqrt{y_{2}^{2}+2 g\left(z_{* 2}-z_{* 1}\right)}}=0 ; \\
\frac{d y_{2}}{d t}=\frac{2 g\left(z_{* 2}-z_{* 1}\right) \mathrm{B}}{y_{2}\left[\sqrt{y_{2}^{2}+2 g\left(z_{* 2}-z_{* 1}\right)}-y_{2}\right]} .
\end{gathered}
$$

Аналитическое решение системы уравнений (35) имеет вид:

$$
\begin{gathered}
\alpha_{11}=\frac{\rho_{0}}{s_{*}} \mathrm{~B}(\mathrm{~A}+\mathrm{B} t) t ; \quad \alpha_{21}=-\rho_{0} g \frac{\mathrm{B}}{\mathrm{A}} t ; \\
\alpha_{12}=\alpha_{11}(\Delta \tau) \frac{y_{2}+y}{y_{2 \tau}+y_{\tau}}-\frac{\rho_{0}}{6 g s_{*}\left(z_{* 2}-z_{* 1}\right)}\left(y_{2}+y\right) \Gamma ; \\
\alpha_{22}=\alpha_{21}(\Delta \tau) \frac{y_{2}+y}{y_{2 \tau}+y_{\tau}}-\frac{\rho_{0}}{2\left(z_{* 2}-z_{* 1}\right)}\left(y_{2}+y\right)\left\{y_{\tau}-y-\frac{\Gamma}{3 g\left(z_{* 2}-z_{* 1}\right)}\right\}
\end{gathered}
$$




$$
\begin{gathered}
\alpha_{3}=\frac{\rho_{0}}{2\left(z_{* 2}-z_{* 1}\right)}\left(y_{2 \tau}-y_{2}\right)\left(y_{2}-\mathcal{Y}\right) ; \\
\alpha_{4}=\alpha_{11}(\Delta \tau) \frac{s_{*}}{\Delta \tau} \cdot \frac{y_{2}+\mathcal{Y}}{y_{2 \tau}+\mathcal{Y}_{\tau}} ; \\
y=\sqrt{y_{2}^{2}+2 g\left(z_{* 2}-z_{* 1}\right)} ; \quad y_{2 \tau}=\sqrt{2 g\left(z_{* 2}-z_{* 1}\right)+(\mathrm{A}+\mathrm{B} \cdot \Delta \tau)^{2}} ; \\
\Gamma=-y_{2}^{3}+y_{2 \tau}^{3}+\mathcal{Y}^{3}-\mathcal{Y}_{\tau}^{3} .
\end{gathered}
$$

Соотношения (36) совместно с соотношениями, получаемыми из условия минимума целевой функции, дают замкнутую систему четырех нелинейных уравнений для определения параметров $S_{*}, Z_{* 1}, Z_{* 2}, \Delta \tau$ двух последовательно возникающих утечек, решение которой может служить тестом

$$
\begin{aligned}
& \sum_{n=1}^{N} \alpha_{j n} \cdot\left(\varphi_{n}-p_{1 n}\right)=0, \quad J=1,2,3,4, \\
& \alpha_{i}=\left\{\begin{array}{cc}
\alpha_{i 1} & 1 \leq n \leq n_{\tau} \\
\alpha_{i 2} & n_{\tau}<n \leq N
\end{array}, \quad i=1,2 .\right.
\end{aligned}
$$

Громоздкость этих уравнений не дает возможности указать явное аналитическое выражение параметров утечки. Величина погрешности их восстановления определяется численно после завершения итераций по соотношениям, аналогичным (19), (20). Наиболее простым является вариант, когда время образования второй утечки превышает интервал времени, необходимый для идентификации параметров первой утечки.

\section{Утечка на фоне плановой откачки}

Если рассматривать динамику развития первой утечки (согласно полученному решению) как некоторый технологический процесс, то появление второй утечки можно интерпретировать как разладку в 
«нормальном» протекании процесса и использовать статистический метод установления ее параметров. «Псевдостохастичность» процесса может быть получена путем внесения в (точные) измерения ошибок, распределенных по различным законам (нормальному, равномерному, квадратично-нормальному, Лапласа и др.).

Первые работы, посвященные задаче максимально быстрого обнаружения изменений в процессах, имеющих вероятностный характер, относятся к 30-м годам прошлого века. В 1959 г. А.Н. Колмогоров и А.Н. Ширяев предложили формальную постановку задачи «последовательного обнаружения спонтанно возникающих эффектов», которая может интерпретироваться как задача об «утечке». А.Н. Ширяев нашел оптимальное решение этой задачи в случае полной априорной информации о функции распределения наблюдений и момента «утечки» [10]. В работах $[11,12]$ сформулирована проблема, относящаяся и к задаче обнаружения «утечек» в трубопроводах. Установлено [13], что задачу обнаружения «утечки» необходимо рассматривать при различных законах (отличных от нормального) распределения контролируемой характеристики, например интенсивности второй утечки. Особое значение эта проблема имеет для выявления несанкционированных отборов жидкости. Алгоритм расчета утечки на основе статистического метода [14] также может быть протестирован на рассматриваемых аналитических решениях, но рассмотрение этого вопроса выходит за рамки данной работы.

\section{Утечка через отверстие с переменной во времени площадью}

Решение уравнения (11) легко получить в виде квадратур и при условии, что $s_{*}$ является функцией времени вида:

$$
S_{*}=S_{* 0}\left\{\begin{array}{lr}
\psi, & 0 \leq t<t_{*} \\
1, & t_{*} \leq t
\end{array}\right.
$$


где $\psi=\psi(t)$ - произвольная функция времени, обращающаяся в нуль при $t=0$

$t_{*}-$ верхняя граница времени раскрытия отверстия утечки.

После разделения переменных интегральную кривую, проходящую через нулевую точку, можно записать следующим образом:

$$
\begin{gathered}
p_{d}=p_{a t m}+\rho_{0} g\left(z_{*}-z_{d}\right)+\frac{\rho_{0}}{2}[\mathrm{~A}+\mathrm{B} \bar{\psi}]^{2} \\
\bar{\psi}=\left\{\begin{array}{cc}
\int_{0}^{t_{*}} \psi d t & 0 \leq t<t_{*} \\
t & t_{*} \leq t
\end{array}\right.
\end{gathered}
$$

При непрерывном линейном законе раскрытия $\left(\psi\left(t_{*}\right)=1\right)$ отверстия утечки функция $\bar{\psi}$ принимает вид:

$$
\bar{\psi}=\left\{\begin{array}{cc}
\frac{t^{2}}{2 t_{*}} & 0 \leq t<t_{*} \\
t & t_{*} \leq t
\end{array} .\right.
$$

При таком законе моменту полного раскрытия отверстия будет соответствовать разрыв производной второго порядка функции $p_{d}$ по времени. Можно построить решение с разрывом первой производной, для этого достаточно задать с помощью дельта функции Дирака в точке $t<t$ * разрыв в законе раскрытия. Скачок давления можно получить, вводя разрыв функции $\bar{\psi}$, но физическая интерпретация такого варианта в рамках принятой модели истечения затруднительна. Определенный интерес представляет вопрос построения функции $\bar{\psi}$, вносящей минимальное возмущение в исходный (фоновый) нестационарный процесс. A priori можно утверждать, что малые утечки с непрерывным законом раскрытия будут наиболее трудны для идентификации как любым параметрическим методом, так и большинством алгоритмов статистического метода. 
Варианты такого типа особо предпочтительны для тестирования алгоритмов идентификации несанкционированных отборов. Примером такого закона раскрытия является производная от функции $\bar{\psi}$, когда она равна разности экспоненты в отрицательной степени, и ее разложения в ряд Маклорена:

$$
\begin{gathered}
\bar{\psi}=\exp (-\widetilde{\beta} t)-1+\widetilde{\beta} t-\frac{\widetilde{\beta}^{2}}{2 !} t^{2} \ldots+(-1)^{2 n} \frac{\widetilde{\beta}^{2 n-1}}{(2 n-1) !} t^{2 n-1}-(-1)^{2 n} \frac{\widetilde{\beta}^{2 n}}{(2 n) !} t^{2 n} \\
s_{*}=s_{* 0} \frac{d \bar{\psi}}{d t} .
\end{gathered}
$$

Началу раскрытия в этом случае сопутствует возмущение в производных функции давления с порядком выше $(2 n-1)$, обнаружить которые волновые методы не в состоянии. Параметр $\widetilde{\beta}$ или $t_{*}$ можно присоединить к другим параметрам утечки и проводить анализ возможностей того или иного «не волнового» алгоритма идентификации.

\section{Отбор жидкости в герметичную емкость, заполненную газом}

Уравнение, описывающее процесс свободного истечения, в этом случае также может быть получено из (11) путем замены величины $p_{a t m}$ функцией противодавления $p_{\Lambda}$. Величина $p_{\Lambda}$ определяется из условий неизменности массы газа в объеме газовой полости и политропичности процесса ее сжатия. После очевидных преобразований расчетные уравнения приобретают вид:

$$
\frac{\mathrm{d}}{\mathrm{dt}} p_{d}=-\frac{\mu \rho_{0} c^{2}}{L f_{0}} s_{*} \cdot \sqrt{2 g \zeta_{p}\left[z_{d}-z_{*}+\frac{p_{d}-p_{\Lambda}}{\rho_{0} g}\right]},
$$




$$
\frac{p_{d}-p_{01}}{c^{2}} L f_{0}+\rho_{0} \Lambda_{0}\left(1+\frac{p_{\Lambda}-p_{0}}{\mathrm{~K}_{g}}\right)\left[1-\left(\frac{p_{\Lambda}}{p_{a t m}}\right)^{-\frac{1}{\chi}}\right]=0
$$

здесь $\Lambda_{0}-$ объем емкости;

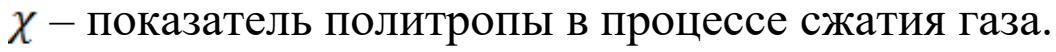

Подстановка $p_{d}$, определяемого из второго соотношения, в первое уравнение приводит его к дифференциальному уравнению, решение которого в форме квадратуры имеет вид:

$$
\int_{p_{a t m}}^{p_{\Lambda}}\left(2 g \zeta_{p}\left[z_{d}-z_{*}+\frac{p_{d}-p_{\Lambda}}{\rho_{0} g}\right]\right)^{-0.5}\left\{1-\left(1-\frac{\mathrm{K}_{g}}{\chi} \frac{1}{p_{\Lambda}}\right)\left(\frac{p_{\Lambda}}{p_{a t m}}\right)^{-\frac{1}{\chi}}\right\} d p_{\Lambda}=\frac{\mu s_{*} \mathrm{~K}_{g}}{\Lambda_{0}} t .
$$

Это решение может быть применено как для покоящейся жидкости, так и на фоне нестационарного процесса от первой утечки. Следует отметить, что тесты с желательными «особенностями» кривой давления можно строить, предполагая, что параметр $p_{a t m}$ является произвольной функцией. Этот произвол можно использовать в целях тестирования алгоритмов идентификации вне зависимости от физического смысла такой функции.

В заключение укажем тест для идентификации параметров источника гармонического возмущения, находящегося в сечении утечки.

Примем, что возмущение от внешней среды (противодавление) определяется функцией $\Phi(t)$ следующего вида:

$$
\Phi(t)=p_{a t m}+\varepsilon_{\Delta} \sin \omega t+\frac{1}{2 \rho_{0} \mathrm{~B}^{2}}\left[\varepsilon_{\Delta}^{2} \omega^{2} \cos ^{2} \omega t+2 \rho_{0} \mathrm{~B} \varepsilon_{\Delta} \omega(\mathrm{A}+\mathrm{B} t) \cos \omega t\right]
$$

Легко проверить, что давление в сечении нахождения датчика определяется функцией:

$$
\mathcal{P}_{d}=\bar{p}_{d}+\varepsilon_{\Delta} \sin \omega t=p_{a t m}+\rho_{0} g\left(z_{*}-z_{d}\right)+\frac{\rho_{0}}{2}(\mathrm{~A}+\mathrm{B} t)^{2}+\varepsilon_{\Delta} \sin \omega t,
$$


здесь $\bar{p}_{d}-$ решение при истечении в неограниченную среду с атмосферным давлением $\Phi(t) \equiv p_{a t m}$.

На рисунке 7 приведены графики функции $\Phi$, относящегося к нему давления $\mathcal{P}_{d}$, а также график функции $\bar{p}_{d}$ для вариантов а) и б) соответственно с различными одинаковыми высотными расположениями датчика и сечения утечки. Параметры $\varepsilon_{\Delta}, \omega$ выбраны таким образом, чтобы решение прямой задачи имело простую физическую интерпретацию, которая возможна при условии $\Phi \geq p_{s}$; $\left(p_{s}-\right.$ давление насыщенных паров). Особенностью рассматриваемого теста является возможность регулирования величины разрыва первой производной, а также получения аналитических зависимостей, связывающих параметры утечки с «точными» измерениями. Практически очевидный характер влияния противодавления на «точно измеряемое» давление наглядно иллюстрируется на рисунке 7.
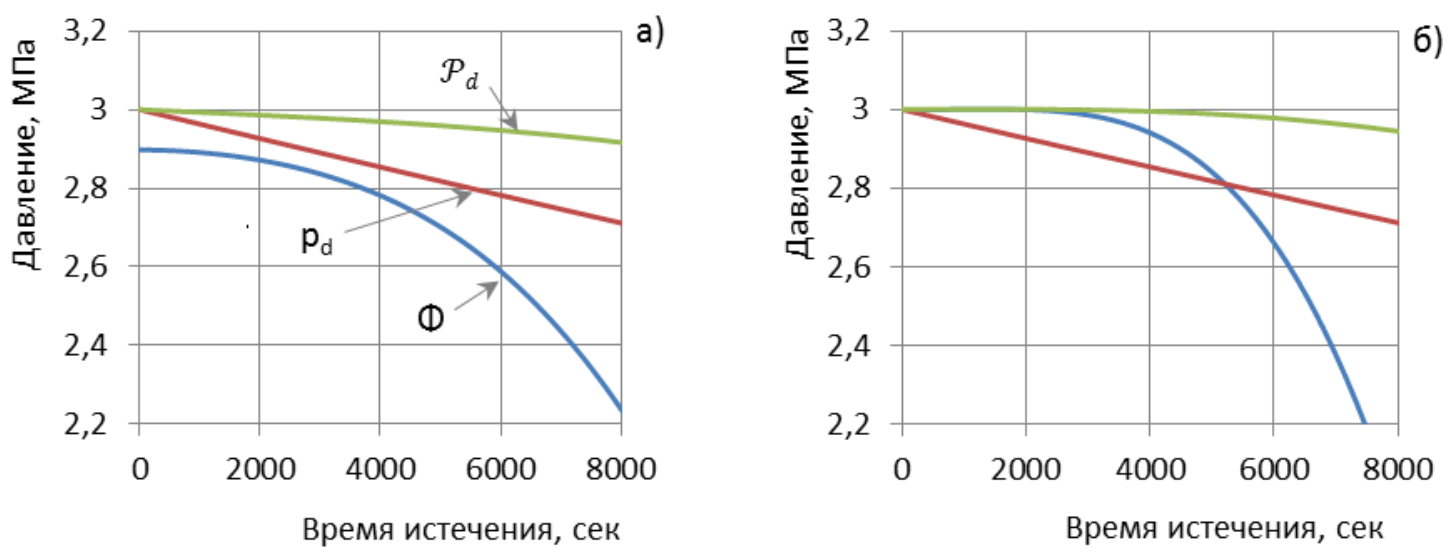

a) $\left(\mathrm{z}_{\mathrm{d}}-\mathrm{Z}^{*}\right)=20 \mathrm{M}$;

б) $\left(\mathrm{z}_{\mathrm{d}}-\mathrm{Z}^{*}\right)=0$

Рисунок 7. Влияние давления во внешней среде 
Функции чувствительности $\alpha_{1}, \alpha_{2}$ остаются без изменения, к ним добавляются функции чувствительности $\alpha_{3}, \alpha_{4}$ по параметрам $\varepsilon_{\Delta}, \omega$ соответственно: $\alpha_{3}=\sin \omega t ; \alpha_{4}=\varepsilon_{\Delta} \cdot t \cos \omega t$.

Расчетные уравнения для параметров $Z_{*}, Z_{*}, \varepsilon_{\Delta}, \omega$, как и ранее, получают из условия минимума целевой функции. Построение же и анализ алгоритма решения получаемых при этом уравнений выходит за рамки данной статьи.

Необходимо отметить, что все рассматриваемые в данной работе тесты характеризуются одним существенным условием: $\mathrm{p}\left(z_{\max }, t\right)>p_{a t m}$. При нарушении этого условия во внутреннем объеме трубопровода происходит переход к двухфазной структуре среды. В зависимости от характера «высотного» профиля трубопровода, а также взаимного расположения датчика и утечки реализуются различные варианты протекания дальнейшего процесса, которые требуют специального рассмотрения. Очевидно, что в этом случае наличие нескольких датчиков будет играть, существенную роль для идентификации (в отличие от рассмотренного нами варианта, в котором анализируется лишь один временной ряд).

Аналитические выражения погрешности восстановления характеристик утечки и параметрические расчеты показали, что рассматриваемый метод дает обнадеживающие результаты во всех случаях, в том числе и в вариантах с развитием утечки по практически безволновому сценарию. Длительность интервала идентификации приводит к необходимости учета тепловых потерь при рассмотрении реального процесса. Для этого достаточно присоединить к уравнению баланса масс уравнение теплового баланса [15], учесть влияние неизотермичности на зависимости (1), (2). Процедура тестирования метода решения обратной задачи при этом остается неизменной. 


\section{Выводы}

Исследован процесс восстановления параметров утечки (обратная задача) с помощью метода функций чувствительности. Основной вариант обратной задачи решен полностью аналитически, часть вариантов доведена до системы конечных нелинейных соотношений.

Исследовано поведение метода и реализующего его алгоритма в случаях, когда измерения отличаются от идеальных. Для этого в исходное аналитическое решение внесены различного вида возмущения. Рассмотрены такие факторы, как величина площади отверстия (расход) утечки, высотная отметка сечения утечки, величина временного интервала, на котором осуществляется идентификация, системные ошибки и периодические возмущения замеров давления в пределах погрешности датчиков, а также алгоритмы предварительной обработки исходной информации.

Получено аналитическое представление погрешности восстановления параметров утечки и рассмотрены особенности сходимости алгоритма. Установлено существование минимально необходимого временного интервала, начиная с которого идентификация по рассматриваемому методу становится возможной, а также существование пределов достижимой точности идентифицированных параметров.

Приведено несколько тестов, позволяющих получать решение некоторых обратных задач в аналитической форме. Отличительные особенности предлагаемых тестов заключаются в возможности получения аналитических выражений погрешности одновременного восстановления нескольких (более двух) параметров утечки. 


\section{Список используемых источников}

1. Численные методы в задачах физики быстропротекающих процессов / А.В. Бабкин, В.И. Колпаков, В.Н. Охитин, В.В. Селиванов. 2-е изд., испр. М.: Изд-во МГТУ им. Н.Э. Баумана, 2006. 520 с.: ил.

2. Гамзаев X.M. Некоторые задачи трубопроводного транспорта нефти и нефтепродуктов // Электронный научный журнал «Нефтегазовое дело». 2007. № 2. URL: http://ogbus.ru/authors/Hamzayev/Hamzayev_1.pdf (дата обращения: 05.02.2018)

3. Авдюшев В.А., Мезенцева А.Д. Метод наименьших модулей и его эффективность при обработке измерений с ошибками различного распределения // Известия вузов. Физика. 2012. Т. 55. № 10/2. С. 68-76.

4. Воеводин А.Ф., Никифоровская В.С. Численный метод определения места утечки жидкости или газа в трубопроводе // Сиб. журн. индустр. матем. 2009. Т. 12, № 1. С. 25-30.

5. Трубопроводный транспорт нефти: в 2 т. / С.А. Бакшина и др.; под ред. С.М. Вайнштока. М.: ООО «Недра-Бизнесцентр», 2004. Т. 2: 621 с.

6. Воеводин А.Ф., Никифоровская В.С. Численный метод решения некоторых обратных задач гидравлики // Водные ресурсы. 1981. № 3. C. 114-118.

7. Беллман Р., Калаба Р. Квазилинеаризация и нелинейные краевые задачи: пер. с англ. М.: Мир, 1968. 186 с.

8. Бондарев Э.А., Воеводин А.Ф. Методы идентификации нестационарных математических моделей теории фильтрации // Наука и Образование. 2015. № 1. С. 78-82.

9. Теория управления / А.А. Алексеев, Д.Х. Имаев, Н.Н. Кузьмин, В.Б. Яковлев. СПб.: Изд-во СПбГЭТУ «ЛЭТИ», 1999. 435 с.

10. Ширяев А.Н. Статистический последовательный анализ. М.: Наука, 1976. 272 c. 


\section{1. Томашевский}

A.В., Снежной

Г.В.

Использование последовательного критерия Вальда для обнаружения «разладок» технологических операций // Авиационно-космическая техника и технология. 2008. № 10 (57). С. 222-226.

12. Салов Г.И. Задача о разладке для скачкообразного марковского процесса // Индустриальная математика. 2008. Т. 11, № 1. С. 116-121.

13. Сунагатуллин Р.3., Коршунов С.А., Дацов Ю.В. К вопросу технического и методологического сопровождения систем обнаружения утечек // Наука и технологии трубопроводного транспорта нефти и нефтепродуктов. 2017. Т. 7. № 5. С. 42-50.

14. Сухарев М.Г., Косова К.О., Титов Б.А., Чудина М.Ю. Стохастическая модель идентификации утечек // Наука и технологии трубопроводного транспорта нефти и нефтепродуктов. 2015. № 4. С. 80-84.

15. Гинзбург И.П. Прикладная гидрогазодинамика. Л.: Изд-во Ленинградского университета, 1958. 338 с.

\section{References}

1. Babkin A.V., Kolpakov V.I., Okhitin V.N., Selivanov V.V. Chislennye metody $v$ zadachakh fiziki bystroprotekayushchikh protsessov [Numeracal Methods in Physics Problems of Fast Processes]. 2nd ed. Moscow, Izd-vo MGTU im. N.E. Baumana, 2006. 520 p. [in Russian].

2. Gamzaev Kh.M. Nekotorye zadachi truboprovodnogo transporta nefti i nefteproduktov [Some Tasks of Pipeline Transportation of Crude Oil and Petroleum Products]. Elektronnyi nauchnyi zhurnal «Neftegazovoe delo»Electronic Scientific Journal «Oil and Gas Business», 2007, No. 2. URL: http://ogbus.ru/authors/Hamzayev/Hamzayev_1.pdf (accessed 05.02.2018). [in Russian]. 
3. Avdyushev V.A., Mezentseva A.D. Metod naimen'shikh modulei i ego effektivnost' pri obrabotke izmerenii s oshibkami razlichnogo raspredeleniya [Least Absolute Deviation Method and its Efficiency in Working with Error Measurements of Different Origin]. Izvestiya vuzov. Fizika - Russian Physics Journal, 2012, Vol. 55, No. 10/2, pp. 68-76. [in Russian].

4. Voevodin A.F., Nikiforovskaya V.S. Chislennyi metod opredeleniya mesta utechki zhidkosti ili gaza $\mathrm{v}$ truboprovode [Numerical Method of Oil or Gas Leakage Place Identification in the Pipeline]. Sibirskii zhurnal industrialnoi matematiki - Siberian Magazine of Industrial Mathematics, 2009, Vol. 12, No. 1, pp. 25-30. [in Russian].

5. Bakshina S.A. e.a. Truboprovodnyi transport nefti: $v 2 t$. [Oil Pipeline Transport: In 2 Vol.]. Moscow, OOO «Nedra-Biznestsentr» Publ., 2004. Vol. 2: 621 p. [in Russian].

6. Voevodin A.F., Nikiforovskaya V.S. Chislennyi metod resheniya nekotorykh obratnykh zadach gidravliki [Numerical Method of Solving Some Inverse Hydraulic Problems]. Vodnye resursy - Water Resourses, 1981, No. 3, pp. 114-118. [in Russian].

7. Bellman R., Kalaba R. Kvazilinearizatsiya i nelineinye kraevye zadachi: Per. s angl. [Quasilinearization and Nonlinear Boundary Value Problems: Transl. from Engl]. Moscow, Mir Publ., 1968. 186 p. [in Russian].

8. Bondarev E.A., Voevodin A.F. Metody identifikatsii nestatsionarnykh matematicheskikh modelei teorii fil'tratsii [Methods of Identification of Nonstationary Mathematics Modules of Filter Theory]. Nauka i obrazovanie - Science and Education, 2015, No. 1, pp. 78-82. [in Russian].

9. Alekseev A.A., Imaev D.Kh., Kuz'min N.N., Yakovlev V.B. Teoriya upravleniya [Management Theory]. Saint-Petersburg, SPbGETU «LETI»Publ., 1999. 435 p. [in Russian].

10. Shiryaev A.N. Statisticheskii posledovatel'nyi analiz [Sequential Testing]. Mowcow, Nauka Publ., 1976. 272 p. [in Russian]. 
11. Tomashevskii A.V., Snezhnoi G.V. Ispol'zovanie posledovatel'nogo kriteriya Val'da dlya obnaruzheniya «razladok» tekhnologicheskikh operatsii [The Usage of WSPRT (Wald Sequential Probability Ratio Test) for Identification of Technological Breakdowns]. Aviatsionno-kosmicheskaya tekhnika $i$ tekhnologiya - Aerospace Technique and Technology, 2008, No. 10 (57), pp. 222-226. [in Russian].

12. Salov G.I. Zadacha o razladke dlya skachkoobraznogo markovskogo protsessa [Problem of the Step-Rate Markov Process Breakdown]. Industrial'naya matematika - Industrial Mathematics, 2008, Vol. 11, No. 1, pp. 116-121. [in Russian].

13. Sunagatullin R.Z., Korshunov S.A., Datsov Yu.V. K voprosu tekhnicheskogo i metodologicheskogo soprovozhdeniya sistem obnaruzheniya utechek [Concerning Technical and Methodological Support of Leakage Detection Systems at Facilities]. Nauka i tekhnologii truboprovodnogo transporta nefti i nefteproduktov - Science \& Technologies: Oil and Oil Products Pipeline Transportation, 2017, Vol. 7, No. 5, pp. 42-50. [in Russian].

14. Sukharev M.G., Kosova K.O., Titov B.A., Chudina M.Yu. Stokhasticheskaya model' identifikatsii utechek [Stochastic Leaks Identification Model]. Nauka i tekhnologii truboprovodnogo transporta nefti i nefteproduktov - Science \& Technologies: Oil and Oil Products Pipeline Transportation, 2015, No. 4, pp. 80-84. [in Russian].

15. Ginzburg I.P. Prikladnaya gidrogazodinamika [Applied Fluid and Gas Dynamics]. Leningrad, Izd-vo Leningradskogo universiteta, 1958. 338 p. [in Russian]. 


\section{Сведения об авторах}

\section{About the author}

Бондарь Д.В. - заместитель начальника отдела технологических расчетов и моделирования работы магистральных трубопроводов ООО «НИИ Транснефть», г. Москва, Российская Федерация

D.V. Bondar, Deputy Head of Technological Calculations and Simulation Work of Main Pipelines Department, Transneft Research and Development Institute for Oil and Oil Products Transportation (Transneft R\&D), LLC, Moscow, Russian Federation

Жолобов В.В., д-р физ.-мат. наук, ведущий научный сотрудник лаборатории сопровождения программных комплексов ООО «НИИ Транснефть», г. Москва, Российская Федерация

V.V. Zholobov, Doctor of Physical and Mathematical Sciences, Leading Research Associate of Escorts Software Complexes Laboratory, Transneft Research and Development Institute for Oil and Oil Products Transportation (Transneft R\&D), LLC, Moscow, Russian Federation

e-mail: zholobovvv@niitnn.transneft.ru

Варыбок Д.И., ведущий научный сотрудник лаборатории оценки технического состояния ООО «НИИ Транснефть», г. Москва, Российская Федерация

D.I. Varybok, Leading Research Associate of Health Assessment Laboratory, Transneft Research and Development Institute for Oil and Oil Products Transportation (Transneft R\&D), LLC, Moscow, Russian Federation

Надежкин О.C., ведущий научный сотрудник лаборатории технологических расчетов ООО «НИИ Транснефть», г. Москва, Российская Федерация

O.S. Nadezhkin, Leading Research Associate of Technological Calculations Laboratory, Transneft Research and Development Institute for Oil and Oil Products Transportation (Transneft R\&D), LLC, Moscow, Russian Federation 